\title{
Evolution of lithium clusters to superatomic $\mathrm{Li}_{3} \mathrm{O}^{+}$
}

Henri Pauna, Xinying Shi, Marko Huttula, Esko Kokkonen, Taohai Li, Youhua Luo, Jyrki Lappalainen, Meng Zhang, and Wei Cao

Citation: Appl. Phys. Lett. 111, 103901 (2017); doi: 10.1063/1.5001700

View online: http://dx.doi.org/10.1063/1.5001700

View Table of Contents: http://aip.scitation.org/toc/apl/111/10

Published by the American Institute of Physics

\section{Articles you may be interested in}

Power conversion process in magnetoelectric gyrators

Applied Physics Letters 111, 103902 (2017); 10.1063/1.4999371

Uniaxial-stress-driven transformation in cold compressed glassy carbon

Applied Physics Letters 111, 101901 (2017); 10.1063/1.4996278

Response of $\mathrm{LiTaO}_{3}$ thin film to X-band short microwave pulse

Applied Physics Letters 111, 102901 (2017); 10.1063/1.4994964

Flexible and wearable 3D graphene sensor with $141 \mathrm{KHz}$ frequency signal response capability Applied Physics Letters 111, 103501 (2017); 10.1063/1.5001472

Variable range hopping electric and thermoelectric transport in anisotropic black phosphorus

Applied Physics Letters 111, 102101 (2017); 10.1063/1.4985333

Ultra-broadband and planar sound diffuser with high uniformity of reflected intensity

Applied Physics Letters 111, 103502 (2017); 10.1063/1.4993845

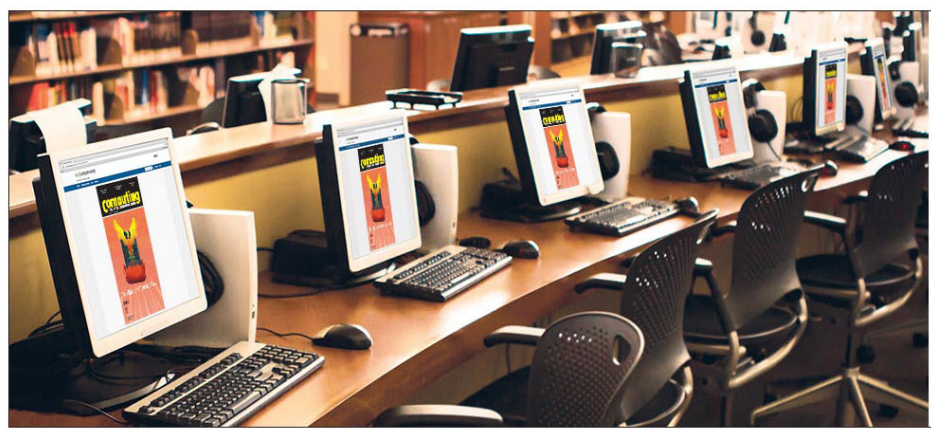

CiSE is already at your fingertips...

comping

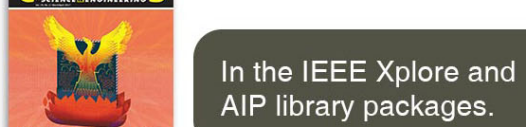

AIP library packages. 


\title{
Evolution of lithium clusters to superatomic $\mathrm{Li}_{3} \mathrm{O}^{+}$
}

\author{
Henri Pauna, ${ }^{1, a)}$ Xinying Shi, ${ }^{1}$ Marko Huttula, ${ }^{1}$ Esko Kokkonen, ${ }^{1}$ Taohai $L i,{ }^{2}$ Youhua Luo, ${ }^{3}$ \\ Jyrki Lappalainen, ${ }^{4}$ Meng Zhang, ${ }^{3, b)}$ and Wei Cao ${ }^{1}$ \\ ${ }^{1}$ Nano and Molecular Systems Research Unit, University of Oulu, P.O. Box 3000, FIN-90014 Oulu, Finland \\ ${ }^{2}$ College of Chemistry, Key Lab of Environment Friendly Chemistry and Application in Ministry of Education, \\ Xiangtan University, Yuhu District, Xiangtan 411105, China \\ ${ }^{3}$ Department of Physics, East China University of Science and Technology, Shanghai 200237, China \\ ${ }^{4}$ Faculty of Information Technology and Electrical Engineering, University of Oulu, P.O. Box 4500, \\ FIN-90014 Oulu, Finland
}

(Received 23 May 2017; accepted 25 August 2017; published online 6 September 2017)

\begin{abstract}
Accurate knowledge of the oxidation stages of lithium is crucially important for developing nextgeneration Li-air batteries. The intermediate oxidation stages, however, differ in the bulk and cluster forms of lithium. In this letter, using first-principles calculations, we predict several reaction pathways leading to the formation of $\mathrm{Li}_{3} \mathrm{O}^{+}$superatoms. Experimental results based on time-of-flight mass spectrometry and laser ablation of oxidized lithium bulk samples agreed well with our theoretical calculations. Additionally, the highest occupied molecular orbital-lowest unoccupied molecular orbital gap of $\mathrm{Li}_{3} \mathrm{O}^{+}$was close to the energy released in one of these reaction paths, indicating that the superatom could act as a candidate charge-discharge unit. Published by AIP Publishing.
\end{abstract}

[http://dx.doi.org/10.1063/1.5001700]

Lithium and its compounds are widely used in energy storage materials. Owing to their electrochemical properties, Li-based composites can act as both a good conductor and a semiconductor; switching of the conductivity can be realized by charging and discharging. For example, semiconducting lithium peroxide ${ }^{1}$ is composed of two equivalent lithium and oxygen atoms. ${ }^{2}$ The formation of this compound is undesirable on the surface of lithium battery cathodes; ${ }^{3}$ however, $\mathrm{Li}_{2} \mathrm{O}_{2}$ composites plays a key role in large volume Li-air batteries. ${ }^{3-8}$ The charge-discharge pathways of $\mathrm{Li}_{2} \mathrm{O}_{2}$ have been extensively studied in the determination of suitable battery electrolytes. ${ }^{3,6,8}$ To further enlarge the charge-to-mass ratio of batteries, nano-and sub-nano-scale charge carriers are considered to be more capable candidates than their bulk counterparts. ${ }^{9}, 10$

In contrast to the numerous studies of $\mathrm{Li}_{2} \mathrm{O}_{2}$ in the bulk phase, investigations of free $\mathrm{Li}-\mathrm{O}$ clusters have mainly focused on hyper-lithiated $\mathrm{Li}_{3} \mathrm{O}^{(0, \pm)}$ clusters. This set of clusters is of particular interest owing to its excess valence electrons. These clusters have been termed "superalkali atoms" in their neutral or charged forms. ${ }^{11-14}$ Although the structure and properties of $\mathrm{Li}_{3} \mathrm{O}$ clusters have been systematically investigated, ${ }^{15}$ their formation processes are not yet fully understood. Furthermore, despite many early studies on the thermochemical properties of free $\mathrm{Li}-\mathrm{O}$ clusters by mass spectrometry, ${ }^{16}$ there has been no physical characterization of lithium bulk oxidation states and those of corresponding cluster formation. As building blocks of many nanomaterials with special functionalities, ${ }^{17,18}$ Li-based clusters also possess unique properties, which could be applied to Li-air batteries. ${ }^{7}$ Thus, studies of the formation mechanisms and transformations between $\mathrm{Li}-\mathrm{O}$ clusters might provide new insights into future energy storage materials.

\footnotetext{
${ }^{\text {a)} E-m a i l: ~ h e n r i . p a u n a @ o u l u . f i ~}$

b)E-mail: mzhang@ecust.edu.cn
}

In this letter, we report on the evolution and transformation of free lithium clusters to superatomic $\mathrm{Li}_{3} \mathrm{O}^{+}$. The stability and reaction pathways of $\mathrm{Li}_{3} \mathrm{O}^{+}$are predicted by firstprinciples calculations and supported by time-of-flight mass spectrometry (TOFMS) experiments. We propose a physical mechanism for the formation of $\mathrm{Li}_{3} \mathrm{O}^{+}$as a competing process to the formation of $\mathrm{Li}-\mathrm{O}$ clusters during the charge-discharge effect. These processes could be the principle, intermediate, or final stages for future nanostructured $\mathrm{Li}$-air batteries.

To identify potential reaction pathways among lithium clusters, first-principles computations were performed via allelectron relativistic spin-unrestricted calculations with the DMOL $^{3}$ program. ${ }^{19}$ The revised Perdue-Burke-Ernzerhof (RPBE) functional ${ }^{20}$ and double numeric plus polarization basis set with addition of diffuse functions (DNP+) were used. Convergence criteria for the self-consistent field (SCF) were set to $1 \times 10^{-6}$ hartree for total energy and electron density, $2 \times 10^{-3}$ hartree $/ \AA$ for the gradient and $5 \times 10^{-3} \AA$ for the displacement. Harmonic vibrational analysis was performed at the same level of theory to examine the stability of the lowest-energy isomers and estimate the zero-point energy (ZPE) corrections.

Regular solid lithium with purity of $>99.9 \%$ was purchased from Sigma-Aldrich for the experiments. Pieces of solid lithium were pressed to form a flat sample over an area of $0.10 \mathrm{~cm}^{2}$ with a thickness of $0.1 \mathrm{~cm}$. The samples were oxidized in air at different exposure times of $1,2,3$, and $5 \mathrm{~min}$. The oxidation process was initiated by scraping the surface of the sample to reveal a non-oxidized layer. After the oxidation exposure, the samples were placed in a vacuum chamber, which was pre-filled with argon gas to minimize oxidation during pumping. The chamber was pumped to a vacuum range of $1.0 \times 10^{-7}-2.0 \times 10^{-6} \mathrm{mbar}$. This pressure was maintained during all measurements.

A commercial MPL-F-266-30 mW Nd:YAG laser from CNI Laser, Inc., was used as the ablation source with a 
PSU-H-FDA power supply. The laser was operated as a passively Q-switched pulsed laser. The repetition rate was set to $8.77 \mathrm{kHz}$ with a single pulse energy of $\mathrm{E}_{\text {pulse }}=4.26 \mu \mathrm{J}$. In addition to photons with a wavelength of $1064 \mathrm{~nm}$, the laser could also produce 532- and 266-nm photons by frequencydoubling the main lasing transition wavelength. The laser beam typically had a Gaussian intensity distribution on the target surface. We detected no influence of the laser beam shape on ion creation, in the broadening of the time-of-flight peaks. The ions created by the laser ablation were collected with Wiley-McLaren TOFMS equipment. ${ }^{21}$ Instrumental details can be found from Ref. 22. The spectrum from each sample was measured for $5 \mathrm{~min}$.

The highly symmetric $\mathrm{D}_{3 h}$ triangle structure with oxygen atoms in the center is the most stable structure for the $\mathrm{Li}_{3} \mathrm{O}^{+}$ cluster according to our geometry optimizations. The reported bond length between the lithium and oxygen atoms of the $\mathrm{Li}_{3} \mathrm{O}$ cation, with the same point group has been reported to be $1.714 \AA$ by Yokoyama et al. ${ }^{15}$ This length is in good agreement with the results of our density functional theory (DFT) calculations, which gave a bond length of $1.732 \AA$. As shown in the aforementioned studies, the $\mathrm{Li}_{3} \mathrm{O}^{+}$cluster has enhanced stability owing to its superatomic properties. The orbital characteristics, illustrated in Fig. 1, show that two valence electrons from the lithium atoms and six valence electrons from the oxygen atom account for the closed-shell configuration of $1 \mathrm{~S}^{2} 1 \mathrm{P}^{6}$ molecular orbitals. Thus, the $\mathrm{Li}_{3} \mathrm{O}^{+}$cluster contains 8 valence electrons and corresponds to a magic species in

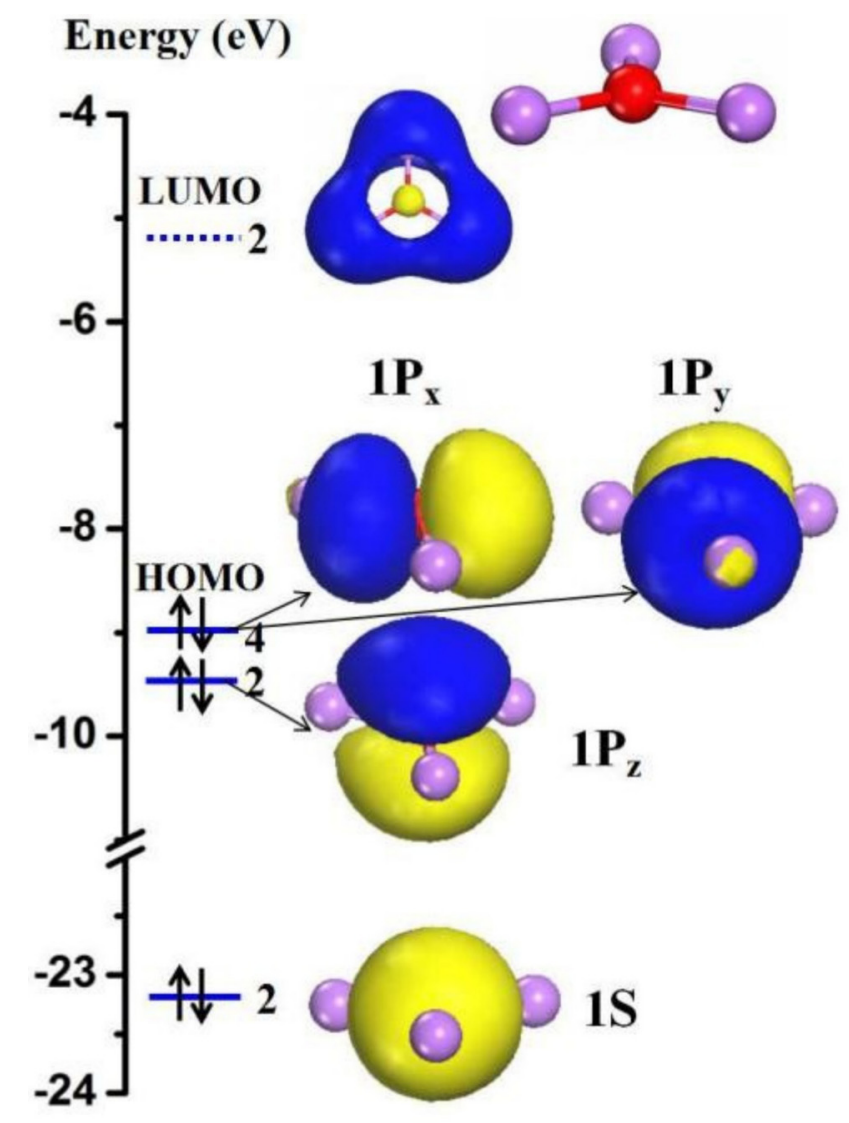

FIG. 1. Molecular orbitals, total molecular orbital energies, and electronic structure of $\mathrm{Li}_{3} \mathrm{O}^{+}$. The highest occupied molecular orbital (HOMO) and lowest unoccupied molecular orbital (LUMO) with the degeneracy indicated. The isovalue is $0.03 \mathrm{e} / \AA^{3}$. accordance with the 18-electron rule. ${ }^{23,24}$ Clearly, the closedshell electron structure is classified as a superatom that consists of specific atoms that share electrons but mimics the chemical behavior of other elements. ${ }^{25-30}$ However, unlike previous reports of magnetic lithium superatoms that were stretched in three dimensions, ${ }^{31} \mathrm{Li}_{3} \mathrm{O}^{+}$possessed a planar structure while maintaining its superatomic properties. As shown in Fig. 1, two electrons occupied the 1S superorbital character, which spread out over the whole $\mathrm{Li}_{3} \mathrm{O}^{+}$cluster. The next orbitals are the two degenerate $1 \mathrm{P}_{z}$ orbitals, while the $1 \mathrm{P}_{x}$ and $1 \mathrm{P}_{y}$ orbitals are degenerate and pushed $0.37 \mathrm{eV}$ higher in energy owing to the planar structure of the $\mathrm{Li}_{3} \mathrm{O}^{+}$ cluster. Thus, $\mathrm{Li}_{3} \mathrm{O}^{+}$has a highest occupied molecular orbital-lowest unoccupied molecular orbital (HOMO-LUMO) energy gap of $3.72 \mathrm{eV}$ according to our DFT calculations and also features enhanced chemical stability.

Possible formation pathways from other lithium clusters to $\mathrm{Li}_{3} \mathrm{O}^{+}$are tabulated in Table I. The clusters on the left side of the reaction pathways are reagents, while $\mathrm{Li}_{3} \mathrm{O}^{+}$on the right side is the final product. The heat release was equal to the difference between the sum of the reagent energies and the total energy of $\mathrm{Li}_{3} \mathrm{O}^{+}$. All these synthesis reactions released energy, indicating a tendency to form the superatoms. The formation processes started with free lithium atoms or dimers as the primary reagents. In (a), the formation of a superatom from $2 \mathrm{Li}+\mathrm{LiO}^{+}$gave the highest energy release of $10.47 \mathrm{eV}$. Thus, this was considered to be the most favorable pathway in the case of neutral gaseous lithium, with a sufficient number of $\mathrm{LiO}^{+}$clusters. Process (b) can be viewed as a variation of (a) where two lithium atoms first form a $\mathrm{Li}_{2}$ dimer, which then reacts with $\mathrm{LiO}^{+}$. Processes (d) and (e) occur when the reactant clusters are $\mathrm{Li}^{0,+}$ and $\mathrm{Li}_{2} \mathrm{O}^{+, 0}$. Less energy was released in processes (d) and (e) than that produced in the processes (a)-(c). This result is unsurprising because in the latter cases the electronegative center of the oxygen has already bonded to two lithium ions and formed a less electronegative $\mathrm{Li}_{2} \mathrm{O}$ or $\mathrm{Li}_{2} \mathrm{O}^{+}$.

Our time-dependent geometry optimizations for processes (a) and (b) clearly showed that those lithium atoms that were not connected to oxygen drifted toward the oxidized compound to form an equilateral triangle with the $\mathrm{D}_{3 h}$ point group. To visualize the formation of the $\mathrm{Li}_{3} \mathrm{O}^{+}$, the processes (a), (b), and (e) in Table I are illustrated in Fig. 2, and animated in supplementary material videos (1), (2), and (3), respectively. In the formation process (a), two individual lithium monomers and the cationic $\mathrm{LiO}^{+}$moved toward each other. The $\mathrm{LiO}^{+}$ion favors moving closer to one of the lithium atoms by rotation of the oxygen atom toward lithium. This interaction with the lithium atom leads to the formation of a linear structure of $\mathrm{Li}_{2} \mathrm{O}^{+}$. Subsequently, a new chemical

TABLE I. Reaction pathways leading to formation of $\mathrm{Li}_{3} \mathrm{O}^{+}$.

\begin{tabular}{lcc}
\hline \hline$\#$ & Formation process & Heat release $E_{\mathrm{h}}(\mathrm{eV})$ \\
\hline (a) & $2 \mathrm{Li}+\mathrm{LiO}^{+} \rightarrow \mathrm{Li}_{3} \mathrm{O}^{+}$ & 10.47 \\
(b) & $\mathrm{Li}_{2}+\mathrm{LiO}^{+} \rightarrow \mathrm{Li}_{3} \mathrm{O}^{+}$ & 9.54 \\
(c) & $\mathrm{Li}_{2}^{+}+\mathrm{LiO} \rightarrow \mathrm{Li}_{3} \mathrm{O}^{+}$ & 6.07 \\
(d) & $\mathrm{Li}^{+} \mathrm{Li}_{2} \mathrm{O}^{+} \rightarrow \mathrm{Li}_{3} \mathrm{O}^{+}$ & 4.51 \\
(e) & $\mathrm{Li}^{+}+\mathrm{Li}_{2} \mathrm{O} \rightarrow \mathrm{Li}_{3} \mathrm{O}^{+}$ & 3.71 \\
\hline
\end{tabular}




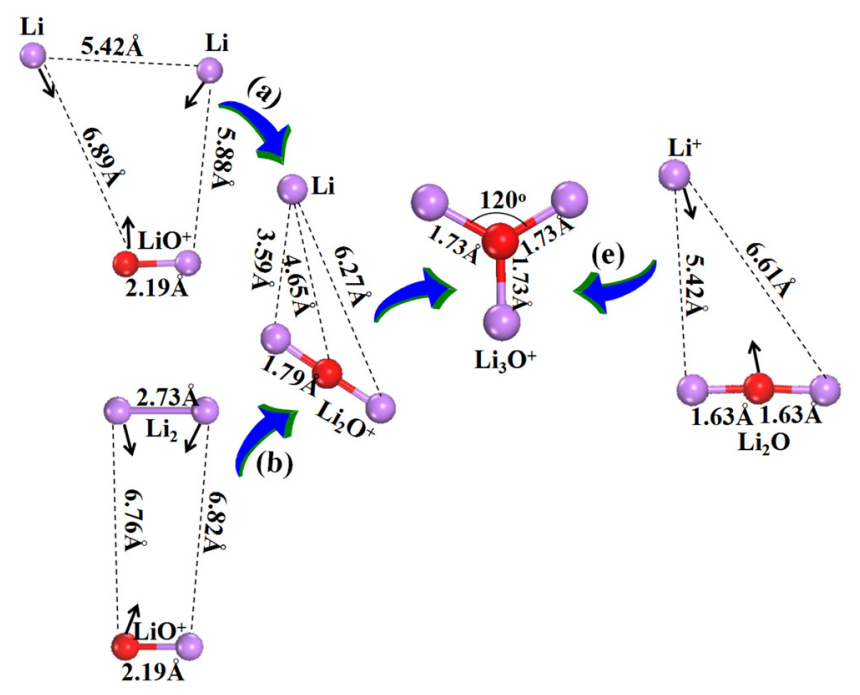

FIG. 2. Formation processes of $\mathrm{Li}_{3} \mathrm{O}^{+}$(a), (b), and (e) from Table I.

bond between another lithium atom and $\mathrm{Li}_{2} \mathrm{O}^{+}$is formed. The $\mathrm{Li}_{3} \mathrm{O}^{+}$superatom is stabilized following the convergence of distances between the oxygen and the surrounding lithium atoms. Process (b) has a similar pathway to that of (a) except that it involves a $\mathrm{Li}_{2}$ dimer instead of two individual lithium atoms. Process (e) can be viewed as the end stage of (a), where the linear $\mathrm{Li}_{2} \mathrm{O}^{+}$starts to bond to the $\mathrm{Li}$ atom. For more detailed optimizations, see supplementary material videos (1), (2), and (3).

To identify lithium clusters, TOFMS measurements of clusters laser-ablated from oxidized lithium samples were performed. The spectra for oxidation times of 1,2,3, and $5 \mathrm{~min}$ are presented in Fig. 3. Mass calibration was performed using the ${ }^{7} \mathrm{Li}^{+},{ }^{7} \mathrm{Li}_{2}^{+}$, and ${ }^{7} \mathrm{Li}_{3}^{+}$peaks. The mass range below 38 amu where the lithium cluster reagents and the final product $\left(\mathrm{Li}_{3} \mathrm{O}^{+}, 37 \mathrm{amu}\right)$ were recorded was studied in more detail. The peaks that are not identified in Fig. 3 were out of the limits of our analysis.

The relative intensities of the peaks varied as a function of oxidation time. For this reason, the time evolution of the oxidized lithium compounds at oxidation times of 1,2, 3, and $5 \mathrm{~min}$ is presented in Table II. The intensities were determined from the integrated TOFMS peak areas. The relative amounts of ${ }^{7} \mathrm{Li}$ and ${ }^{6} \mathrm{Li}$ varied between the spectra. This effect was likely caused by low ion yields, asymmetries in the spectra, and pulsed extraction-field disturbances in the spectrometer. For example, for an oxidation time of $1 \mathrm{~min}$,
TABLE II. Proportion of clusters relative to the integrated mass spectrum signal below $38 \mathrm{amu}(\%)$.

\begin{tabular}{lcccc}
\hline \hline & \multicolumn{4}{c}{ Oxidation time of } \\
\cline { 2 - 5 } Ion & 1 min & 2 min & 3 min & 5 min \\
\hline $\mathrm{Li}^{+}$ & 65.0 & 10.8 & 15.0 & 3.5 \\
$\mathrm{Li}_{2}^{+}$ & 0.1 & 7.2 & 12.1 & 1.1 \\
$\mathrm{Li}_{3}^{+}$ & 0 & 3.4 & 1.8 & 0 \\
$\mathrm{LiO}^{+}$ & 0 & 5.6 & 6.6 & 0 \\
$\mathrm{Li}_{2} \mathrm{O}^{+}$ & 0 & 5.2 & 2.0 & 0 \\
$\mathrm{Li}_{2} \mathrm{O}^{2+}$ & 0 & 1.7 & 1.6 & 0 \\
$\mathrm{Li}_{3} \mathrm{O}^{+}$ & 7.7 & 11.1 & 4.5 & 13.3 \\
\hline \hline
\end{tabular}

the relative abundances of ${ }^{7} \mathrm{Li}$ and ${ }^{6} \mathrm{Li}$ were $91.75 \%$ and $8.25 \%$, respectively, and for $5 \mathrm{~min}$ the values were $93.92 \%$ and $6.08 \%$. Notably $\mathrm{Li}_{3}$ and $\mathrm{LiN}$ molecules have the same mass. However, Li-N compounds are typically formed during combustion rather than slow nitridation in air. Thus, signals from Li-N compounds in the spectra were considered to be negligible.

The $\mathrm{Li}_{3} \mathrm{O}^{+}$superatoms appeared on the samples oxidized for $1 \mathrm{~min}$, and were present in the spectra with longer oxidation times. The reasons for the variation in ion abundance may be attributed to free cluster formation or direct ablation of the lithium sample. However, the existence of $\mathrm{Li}_{3} \mathrm{O}$ in the bulk form has not been revealed in the aforementioned references. Thus, we assumed that its formation occurred through other clusters. This assumption is in line with the TOFMS results. In Fig. 3, many reagent ions in Table I were present in the spectra of samples oxidized for 2 and $3 \mathrm{~min}$. At an oxidation time of $5 \mathrm{~min}$, only the reagents $\mathrm{Li}^{+}$and $\mathrm{Li}_{2}^{+}$were visible, and the majority of the observed ions were $\mathrm{Li}_{3} \mathrm{O}^{+}$clusters.

The lithium samples were also exposed to oxidation times longer than $5 \mathrm{~min}$; however, the total ion yields decreased rapidly even if laser ablation was applied for $30 \mathrm{~min}$. This was most likely because of the formation of very stable $\mathrm{Li}_{2} \mathrm{O}_{2}$ species. It might be possible to study oxidation times beyond $5 \mathrm{~min}$ with a different laser of higher intensity and energy.

The experimental verification of the presence of $\mathrm{Li}_{3} \mathrm{O}^{+}$ in the spectra further suggests that $\mathrm{Li}_{3} \mathrm{O}^{+}$clusters could be applied in energy storage materials. The energy of $3.71 \mathrm{eV}$ released in process (e) is coincidentally close to the $3.72-\mathrm{eV}$ HOMO-LUMO gap of the cluster. This may lead to excitations in nanostructured battery materials involving $\mathrm{Li}_{3} \mathrm{O}^{+}$.

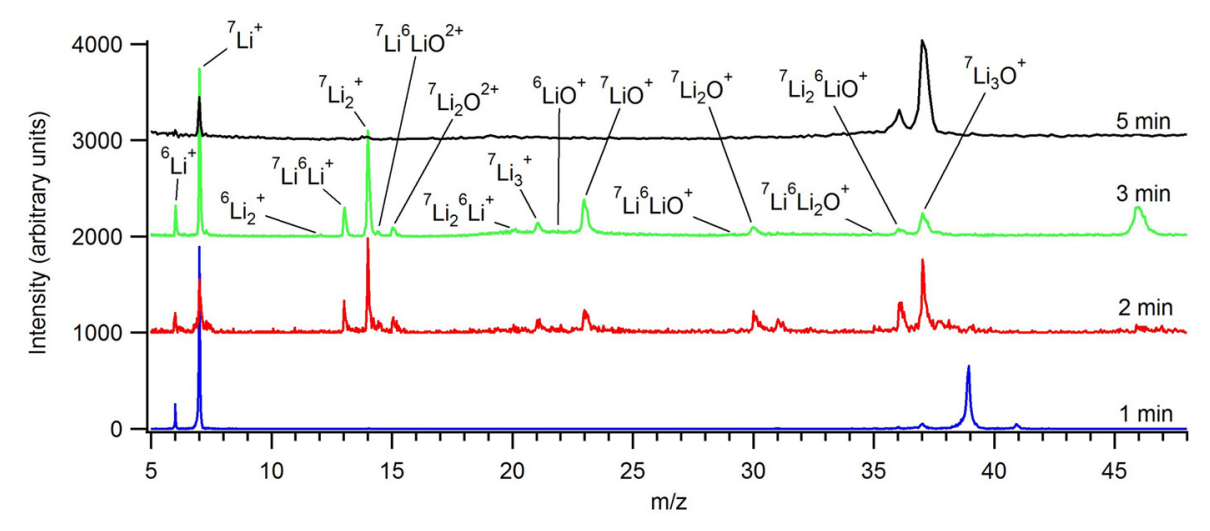

FIG. 3. Spectra for oxidation times of 1 (blue), 2 (red, intensity is multiplied by 10 ), 3 (green), and 5 (black) $\mathrm{min}$. 
When the formation of $\mathrm{Li}_{3} \mathrm{O}^{+}$occurs via process (e), an electron in the $\mathrm{HOMO}$ of an existing $\mathrm{Li}_{3} \mathrm{O}^{+}$could be excited by this exothermic energy. The excitation of $\mathrm{Li}_{3} \mathrm{O}^{+}$followed by relaxation is expressed as

$$
\mathrm{Li}^{+}+\mathrm{Li}_{2} \mathrm{O}+\mathrm{Li}_{3} \mathrm{O}^{+} \rightleftharpoons \mathrm{Li}_{3} \mathrm{O}^{+}+\mathrm{Li}_{3} \mathrm{O}^{+*},
$$

which includes process (e) in Table I. In (1), the storage of energy occurs from left to right (excitation) and release of energy from right to left (relaxation). The stable nature of superatomic $\mathrm{Li}_{3} \mathrm{O}^{+}$might enable its storage in certain media, while excitation, relaxation, and decomposition processes offer a large charge volume for the battery based on the species itself as the charge/discharge unit.

In conclusion, we have performed a thorough investigation of cluster formation pathways leading to stable $\mathrm{Li}_{3} \mathrm{O}^{+}$. The formation processes were further clarified by mass spectroscopy of laser-ablated oxidized lithium bulk samples. The final product of the oxidation process of lithium in the ionic cluster level was the $\mathrm{Li}_{3} \mathrm{O}^{+}$superatom rather than $\mathrm{Li}_{2} \mathrm{O}_{2}$ in the bulk form. The unique structure and the HOMO-LUMO gap energy of $\mathrm{Li}_{3} \mathrm{O}^{+}$make this cluster a possible building block for an energy storage unit. It is hoped that this work will serve as a starting point for the realization of nanostructured energy storage sources for next-generation Li-air batteries.

See supplementary material for videos (1), (2), and (3) for optimization of processes (a), (b), and (e), respectively, in Table I for $\mathrm{Li}_{3} \mathrm{O}^{+}$.

The authors acknowledge financial support from Strategic Grant of Oulu University, the Research Council for Natural Sciences and Engineering of Academy of Finland, and the European Union Regional Development Foundation and Council of Oulu Region. The work was also partially supported by Provincial Natural Science Foundation of Hunan, China (2015JJ2138). This work was financially supported partly by the National Natural Science Foundation of China (Grant No. 11204079). X. Shi acknowledges scholarship sponsored by China Scholarship Council. Tuomas Löytynoja at Nano and Molecular Systems Research Unit is acknowledged for helpful discussions. The authors thank Andrew Jackson, Ph.D., from Liwen Bianji,
Edanz Group China (www.liwenbianji.cn/ac), for editing the English text of a draft of this manuscript.

${ }^{1}$ H. Wu, H. Zhang, X. Cheng, and L. Cai, Philos. Mag. 87, 3373 (2007).

${ }^{2}$ L. Andrews and J. Chern, J. Chem. Phys. 50, 4288 (1969).

${ }^{3}$ X. Gao, Y. Chen, L. Johnson, and P. G. Bruce, Nat. Mater. 15, 882 (2016).

${ }^{4}$ K. C. Lau, R. S. Assary, P. Redfern, J. Greeley, and L. A. Curtiss, J. Phys. Chem. C 116(45), 23890 (2012).

${ }^{5}$ S. Y. Kang, Y. Mo, S. P. Ong, and G. Ceder, Chem. Mater. 25(16), 3328 (2013).

${ }^{6}$ C. V. Amanchukwu, M. Gauthier, T. P. Batcho, C. Symister, Y. ShaoHorn, J. M. D'Arcy, and P. T. Hammond, J. Phys. Chem. Lett. 7(19), 3770 (2016).

${ }^{7}$ Y. Yin, C. Gaya, A. Torayev, V. Thangavel, and A. A. Franco, J. Phys. Chem. Lett. 7(19), 3897 (2016).

${ }^{8}$ H.-G. Jung, J. Hassoun, J.-B. Park, Y.-K. Sun, and B. Scrosati, Nat. Chem. 4, 579 (2012).

${ }^{9}$ U. Das, K. C. Lau, P. C. Redfern, and L. A. Curtiss, J. Phys. Chem. Lett. $\mathbf{5}(5), 813$ (2014).

${ }^{10}$ R. S. Assary, K. C. Lau, K. Amine, Y.-K. Sun, and L. A. Curtiss, J. Phys. Chem. C 117(16), 8041 (2013).

${ }^{11}$ S. Chen, H. L. Xu, L. Zhao, and Z. M. Su, J. Mol. Model. 21(8), 209 (2015).

${ }^{12}$ S. Chen, H.-L. Xu, L. Zhao, and Z.-M. Su, Dalton Trans. 43, 12657 (2014).

${ }^{13}$ S. Zein and J. V. Ortiz, J. Chem. Phys. 135, 164307 (2011).

${ }^{14}$ M. Gutowski and J. Simons, J. Phys. Chem. 98(34), 8326 (1994).

${ }^{15}$ K. Yokoyama, H. Tanaka, and H. Kudo, J. Phys. Chem. A 105(17), 4312 (2001).

${ }^{16}$ C. H. Wu, H. Kudo, and H. R. Ihle, J. Chem. Phys. 70, 1815 (1979).

${ }^{17}$ X. Wang, Y. Luo, T. Yan, W. Cao, and M. Zhang, Phys. Chem. Chem. Phys. 19, 6563 (2017)

${ }^{18}$ M. Zhang, Z. Huang, X. Wang, H. Zhang, T. Li, Z. Wu, Y. Luo, and W. Cao, Sci. Rep. 6, 19504 (2016).

${ }^{19}$ B. Delley, J. Chem. Phys. 92, 508 (1990).

${ }^{20}$ B. Hammer, L. B. Hansen, and J. K. Nørskov, Phys. Rev. B 59, 7413 (1999).

${ }^{21}$ W. C. Wiley and I. H. McLaren, Rev. Sci. Instrum. 26, 1150 (1955).

${ }^{22}$ M. Huttula, M. Harkomaa, E. Nõmmiste, and S. Aksela, Nucl. Instrum. Methods Phys. Res., Sect. A 467-468, 1514 (2001).

${ }^{23}$ P. Pyykkö and N. Runeberg, Angew. Chem., Int. Ed. 114, 2278 (2002).

${ }^{24}$ H. Hiura, T. Miyazaki, and T. Kanayama, Phys. Rev. Lett. 86, 1733 (2001).

${ }^{25}$ J. U. Reveles, P. A. Clayborne, A. C. Reber, S. N. Khanna, K. Pradhan, P. Sen, and M. R. Pederson, Nat. Chem. 1, 310 (2009).

${ }^{26}$ L. J. Cheng and J. L. Yang, J. Chem. Phys. 138, 141101 (2013).

${ }^{27}$ X. X. Zhang, Y. Wang, H. Wang, A. Lim, G. Gantefoer, K. H. Bowen, J. U. Reveles, and S. N. Khanna, J. Am. Chem. Soc. 135(12), 4856 (2013).

${ }^{28}$ D. A. Tomalia and S. N. Khanna, Chem. Rev. 116(4), 2705 (2016).

${ }^{29}$ D. E. Bergeron, P. J. Roach, A. W. Castleman, Jr., N. O. Jones, and S. N. Khanna, Science 307, 231 (2005).

${ }^{30}$ Z. Luo and A. W. Castleman, Acc. Chem. Res. 47(10), 2931 (2014).

${ }^{31}$ M. Zhang, J. Zhang, X. Feng, H. Zhang, L. Zhao, Y. Luo, and W. Cao, J. Phys. Chem. A 117(48), 13025 (2013). 